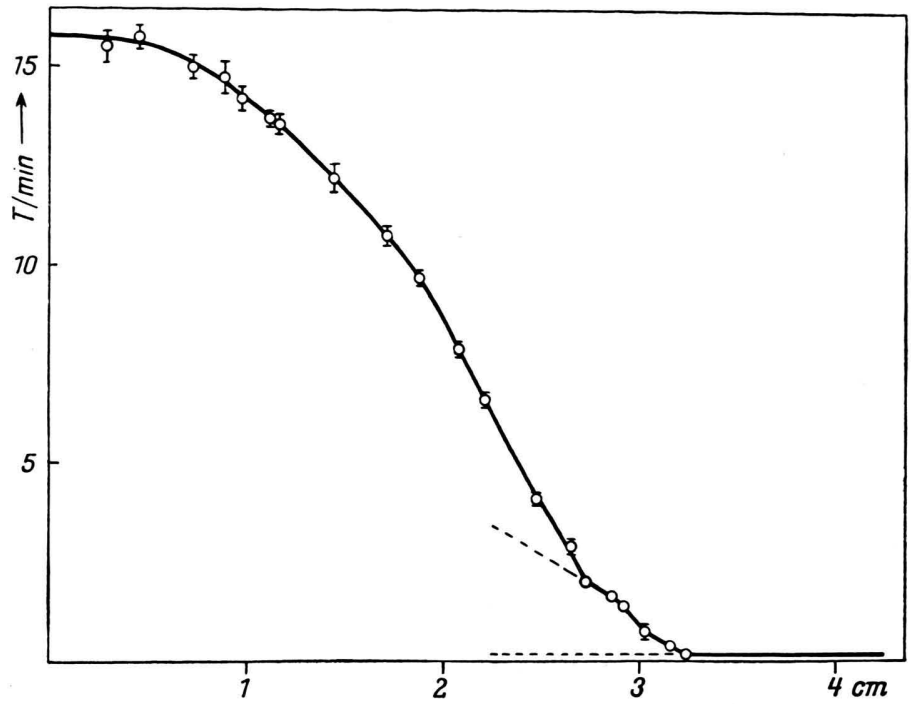

Abb. 3. Reichweitekurve eines Uranpräparates.

Präparates den Nulleffekt zu messen und das störungsfreie Arbeiten des Zählrohrs zu kontrollieren. Der Nulleffekt des Zählrohrs betrug 0,2-0,3 T/min.

Um das zeitraubende und schwierige Einlegen der Aluminiumfolien zu ersparen, wurde der bei A anzusetzende Ansatz später durch einen anderen ersetzt (Abb. 2). Und zwar wurde jetzt auch die Innenfläche des äußeren Aluminiumzylinders als Gleitfläche ausgebildet, auf der ein Messingring nach der gleichen Methode wie der Glaszylinder verschiebbar ist. Auf diesen Ring läßt sich ein Messingzylinder $\mathrm{F}$ aufschrauben. Bei diesem ist der Teil, der vorgeschoben vor dem Zählrohr liegt, bis auf zwei dünne Stege ausgeschnitten, darüber werden zylinderförmig Aluminiumfolien geklebt. Am Kopf des Zylinders ist aus Isolationsgründen noch ein Bernsteinring B, um mit Sicherheit einen Kontakt mit der Zählrohrhochspannung zu vermeiden, angebracht.

Durch diesen neuen Ansatz, der also sowohl die Aluminiumfolien wie auch den früher erwähnten Glaszylinder enthält, wurde eine erhebliche Beschleunigung der Messung erzielt, zudem die Reproduzierbarkeit der Ergebnisse wesentlich verbessert.

Die zu messenden Präparate waren auf Filterpapier aufgebrachte, feinverteilte Fällungen, die in definierter Lage zum Zählrohr auf dem inneren Glaszylinder lagen. Die Aufnahme einer Reichweitekurve erfolgte so, daß jeweils bei einem bestimmten Argondruck zunächst die Zählrohrcharakteristik ermittelt wurde, dann in dem ermittelten Spannungsbereich abwechselnd mit und ohne vorgeschobene Aluminiumfolie gemessen wurde, bzw. durch Vorschieben des Glaszylinders der Nulleffekt kontrolliert wurde.

Es zeigte sich, daß bei räumlich kleinen Präparaten ein Folien-Halbzylinder ausreichend war, d. h. es war dann möglich, auf dem Zylinder 2 Aluminiumfolien aufzubringen und durch Drehen des Flachschliffes einmal mit der einen oder anderen zu messen. Man erhält so für einen eingestellten Druck jeweils drei verschiedene Punkte der Kurve.

Die Bestimmung des Luftäquivalents der Aluminiumfolien erfolgte durch Ausmessen und Wägen. Durch Auswechseln des Folienzylinders konnte mit den verschiedensten Folien gearbeitet werden, ohne die Lage des Präparates zu ändern; die mit verschiedenen Folien gemessenen Kurven stimmten vollkommen überein.

Um das gute Arbeiten mit dem Zählrohr zu zeigen, wurden einige der bekannten $\alpha$-Strahler gemessen. Abb. 3 zeigt die für ein Uranpräparat erhaltene Kurve, in der deutlich die beiden Reichweiten erkennbar sind, sie liegen bei 2,7 und $3,25 \mathrm{~cm}$, was gut mit den anderweitig für Uran bestimmten Werten übereinstimmt ${ }^{2}$. Auch für Sm und Po ergaben sich die aus der Literatur bekannten Werte.

2 S. A. Wytzes u. G. J. van der Maas, Physica 13, 49 [1947].

\section{Massenspektrometrische Bestimmungen an Argon aus Kalisalzen}

Von M. P a h l und J. H i by

Ehemalig. Kaiser-Wilhelm-Institut für Physik, Hechingen

und von F.Smits und W. Gentner

Physikalisches Institut der Universität Freiburg i. Br.

(Z. Naturforschg. 5 a, 404-405 [1950]; eingeg. am 31. Mai 1950)

Wie an anderer Stelle mitgeteilt ${ }^{1}$, sind an Kalisalzen aus Buggingen (Oligozän des Oberrheins) und an einer Sylvinitprobe aus Friedrichshall I (Sehnde bei Hannover) Bestimmungen des Argongehalts durchgeführt worden. Dort finden sich auch genauere Angaben über die Schichten, aus denen die untersuchten Kaliproben stammen. Im folgenden berichten wir über massenspektrometrische Kontrolluntersuchungen der aus den Kalisalzen gewonnenen Argonproben.

Es wird dabei festgestellt, in welchem Ausmaß das aus dem K-Zerfall des Kaliums stammende $40 \mathrm{~A}$ durch Luftargon verunreinigt ist. Hierzu wurde jeweils das Mengenverhältnis der Isotope ${ }^{40} \mathrm{~A} / 36 \mathrm{~A}$ gemessen, das neuerdings $\mathrm{N}$ i e $\mathrm{r}^{2}$ in normalem Luftargon $\mathrm{zu} 296,0 \pm 0,5$ bestimmt hat. Aus den gewonnenen Ergebnissen folgt, inwieweit das gemessene Argon radioaktiven Ursprungs ist. Dadurch soll der natürliche K-Zerfall des Kalium 40 in Argon 40 quantitativ erfaßt und festgestellt werden, unter welchen Bedingungen sich das Kalium-Argon-Verhältnis zu Altersbestimmungen heranziehen läßt.

In einem Arbeitsgang konnten jeweils 200 g Kalisalze verarbeitet werden, die höchstens $6 \mathrm{~mm}^{3}$ Argon liefern. Für die massenspektrometrische Bestimmung wurden mehrere solche Argonproben in einem Röhrchen mit aktiver Kohle gesammelt. Die Isotopenverteilung der Argonprobe

1 F.Smits u. W. Gentner, Geochimica et Cosmochimica Acta, im Erscheinen.

2 A. O. N i e r, Physic. Rev. 77, 789 [1950]. 


\begin{tabular}{|c|c|c|c|c|}
\hline Herkunft des Sylvins & $\mathrm{g} \mathrm{K}$ & $\mathrm{mm}^{3} \mathrm{~A}$ & ${ }^{40} \mathrm{~A} /{ }^{36} \mathrm{~A}$ & $\frac{\mathrm{mm}^{3} \mathrm{rad} \cdot{ }^{40} \mathrm{~A}}{100 \mathrm{~g} \mathrm{~K}}$ \\
\hline $\begin{array}{l}\text { a) Buggingen . . } . . . \\
\text { b) Buggingen, grobkörnig, }>6 \mathrm{~mm} \\
\text { c) wie b), feinkörnig, }<0,3 \mathrm{~mm} \\
\text { d) Buggingen (4 verschiedene) } . \\
\text { e) Friedrichshall (Sehnde) . } \\
\text { f) Luftargon (chemisch gleich behan- } \\
\text { delt wie a-e). . . . . . . }\end{array}$ & $\begin{array}{r}153 \\
243 \\
276 \\
308 \\
54\end{array}$ & $\begin{array}{l}7,4 \\
17,3 \\
12,25 \\
14,7 \\
10,1 \\
\text { ca. } 10\end{array}$ & $\begin{array}{l}3400 \pm 1000 \\
>5000 \\
>3200 \\
1000 \pm 200 \\
1720 \pm 320 \\
293 \pm 9\end{array}$ & $\begin{array}{r}4,4 \pm 0,3 \\
7,1 \pm 0,5 \\
4,5 \pm 0,5 \\
3,25 \pm 0,5 \\
15,5 \pm 1,3\end{array}$ \\
\hline
\end{tabular}

wurde mit einem, ursprünglich von $\mathrm{P}$ a u 13 konstruierten Massenspektrometer untersucht, dessen Ionenquelle abgeändert war und etwa der von Coggeshall und J or d a n 4 beschriebenen entsprach. Aus Intensitätsgründen mußte ein Führungsmagnet für die Elektronen verwendet werden. Der Gasstrom floß aus dem Vorratsgefäß durch einen Strömungswiderstand (molekular) und anschließend durch eine Glasspirale, die mit flüssiger Luft gekühlt war, in die Ionenquelle. Der Eintrittsspalt am Spektrometerrohr hatte die Öffnung $1 \times 0,2 \mathrm{~mm}^{2}$ und diente zugleich als letzter Strömungswiderstand vor der $\mathrm{Hg}$ Diffusionspumpe. Der Gasdruck in der Ionenquelle betrug etwa $10^{-4}$ Torr (gemessen mit Ionisationsmanometer). Die Ionenströme wurden mit einem Elektrometerröhrenverstärker und Galvanometer gemessen, die Ausschläge nach jeder Messung nachgeeicht. Der Gitterableitwiderstand am Eingang des Verstärkers war $1,3 \times 10^{11} \Omega$, der Gitterstrom der Meßröhre T 114 etwa $5 \times 10^{-14}$ Amp. Die Nachweisgrenze lag bei $5 \times 10^{-16} \mathrm{Amp}$. Die Einstellung der Linien erfolgte durch Ändern des Ablenkmagnetfeldes.

Auf allen Massenzahlen von 36 bis 40 fanden sich schwache Störlinien, z. Tl. bis in die Größenordnung $2 \times 10^{-14}$ Amp., auch wenn die Gaszufuhr durch flüssige Luft gekühlt wurde. Die Störlinie 36 nahm erst nach mehrwöchentlicher Betriebsdauer so weit ab, daß ihr Betrag unterhalb der Nachweisgrenze lag. Da anfangs die Störlinie 36 die gleiche Größenanordnung wie die $40 \mathrm{~A}$ Linie bei den Proben aus Kalisalzen aufwies, wurde jeweils der Betrag der Störlinie vor und nach jeder Argonmessung ermittelt und als Korrektur in Abzug gebracht. Die Annahme, daß die Störintensität auch bei Änderung des Gesamtdruckes als additive Konstante betrachtet werden kann, erscheint uns durch das Verhalten anderer, schwacher Verunreinigungslinien gerechtfertigt; wir haben der hierin liegenden Unsicherheit jedoch durch Erhöhung der Fehlergrenze Rechnung getragen. Eine weitere Korrektur war wegen der laufenden Abnahme des Vorratsdrucks erforderlich (Halbwertszeit $126 \mathrm{~min}$ ).

Bisher wurden folgende Ergebnisse erzielt, die in der Tabelle zusammengestellt sind. Die für jede Probe angegebenen g $\mathrm{K}$ entsprechen dem chemischen Analysenwert, der nach der Argonbestimmung gewonnen wurde.

Aus der letzten Spalte der Tabelle ist zu ersehen, daß das Argon-Kalium-Verhältnis nach Korrektur der Luftverunreinigung Schwankungen unterworfen ist, die über den Meßfehler hinausgehen. Für Altersbestimmungen ist

3 W. P a u l, Z. Physik 124, 244 [1948].

4 N. D. C ogg e s hall u. E. B. Jord a n, Rev. sci. Instruments 14, 125 [1943]. daher eine ausführliche Diskussion der geologischen Verhältnisse erforderlich ${ }^{1}$. Vorläufig scheint es berechtigt, den höchsten Argongehalt mit 7,1 $\pm 0,5 \mathrm{~mm}^{3} \mathbf{4 0}^{4} \mathrm{~A} / 100 \mathrm{~g} \mathrm{~K}$ für Altersbestimmungen heranzuziehen. Diese Probe weist die kleinste Luftverunreinigung auf. Salze, in die kein Luftargon hereindiffundiert ist, weisen offenbar auch keinen Argonverlust durch Herausdiffundieren auf.

Aus energetischen Gründen wird die beobachtete $\gamma$ Strahlung dem K-Zerfall zugeordnet. Durch neue massenspektrographische Messungen von Roberts und Nier 5 über die Massendifferenz ${ }^{40} \mathrm{Ca}-40 \mathrm{~A}$ findet diese Zuordnung eine weitere Bestätigung. Die ersten Argonbestimmungen von $\mathrm{Aldrich}$ und $\mathrm{Ni}$ e ${ }^{6}$ an wesentlich älteren Mineralien zeigen, daß die Rate des K-Zerfalls in der gleichen Größenordnung liegt wie die der $\gamma$-Strahlen. Der beste Wert für die Zahl der $\gamma$-Quanten dürfte z. Zt. bei 3,0 Quanten/g K sec liegen 7. Setzt man diesen Wert gleich der Zahl der K-Einfänge, so errechnet sich mit dem obigen Wert von $7,1 \mathrm{~mm}^{3} \mathrm{~A} / 100 \mathrm{~g} \mathrm{~K}$ ein Alter von 20 Millionen Jahren für das Bugginger Lager. Dieser Wert ist in guter Übereinstimmung mit den bisherigen geologischen Altersschätzungen. Es scheinen also beim Kalium keine K-Einfänge ohne begleitende $\gamma$-Strahlung aufzutreten.

Das Salz Friedrichshall I liefert ein wesentlich geringeres Alter, als dem Zechstein (200 Millionen Jahre) zugeordnet wird. Es ist dies wegen der mannigfachen Umlagerung dieser Salze leicht verständlich, doch ist es nicht ausgeschlossen, daß die beschriebene Methode gerade über die Tektonik des Zechsteins interessante Aufschlüsse bringen kann.

5 T. R. R oberts u. A. O. Ni er, briefliche Mitteilung.

${ }_{6}^{6}$ L. T. Aldrich u. A. O. Nier, Physic. Rev. 74, 876 [1949].

7 F. W. S pie rs, Nature [London] 165, 356 [1950].

\section{Zur Plastizität des monoklinen Schwefels}

$$
\text { Von Wolfgang Lorenz }
$$

Physikalisches Institut der Universität Leipzig

(Z. Naturforschg. 5 a, 405-407 [1950]; eingeg. am 26. April 1950)

Der zeitliche Verlauf des Fließens von monoklinem Schwefel wurde in Abhängigkeit von Temperatur und Belastung im Höppler-Konsistometer ${ }^{1}$ untersucht. Bei dieser

1 F. Höppler, Kolloid-Z. 97, 61, 154 [1941]; W. P h ilip of f, Viskosität der Kolloide, Verlag Steinkopff, Dresden 1942, S. 91, 92. 